\title{
Reconstruction of a resected subclavian vein by transposition of the ipsilateral internal jugular vein
}

\author{
Michel Gonzalez, MD, Sébastien Déglise, MD, Hans-Beat Ris, MD, and Jean-Marc Corpataux, MD, \\ Lausanne, Switzerland
}

A heterogeneous group of solid benign and malignant tumors may involve the thoracic inlet. Their surgical management requires complete en bloc resection, sometimes involving major adjacent structures. Generally, the vein is the first invaded element and debate still exists concerning the optimal management. Indeed, owing to the rich collateral circulation, most authors advocate simple ligation after removal of the invaded segment. ${ }^{1}$ Nevertheless, it is sometimes recommended to perform reconstruction owing to the risks of venous hypertension. Herein, we report an interesting reconstruction after subclavian vein resection. A young woman with a thoracic inlet sarcoma invading the left subclavian vein benefited from a superior thoracic wall en bloc resection, with removal of the vein. Venous continuity was restored by transposing the ipsilateral internal jugular vein to the axillary vein.

\section{CLINICAL SUMMARY}

A 27-year-old woman with no relevant comorbidity was admitted to our department for consultation. For the past month, she had been having left upper arm swelling, pain, and incapacitating paresthesis during mobilization. Moreover, a left subclavian $3-\mathrm{cm}$ mass was discovered. A computed tomographic scan (Figure 1) was performed, showing a well-defined tumor between the first rib and the left clavicle. The subclavian vein was invaded and compressed, but the lesion seemed to spare the artery and brachial plexus. Percutaneous core needle biopsy of the mass under ultrasonographic guidance suggested a mesenchymal tumor, but the degree of malignancy remained unclear. Surgical removal was thus recommended, and the patient underwent a left subclavicular approach with en bloc resection with free margins. Resection of the first rib and the clavicle

\footnotetext{
From the Thoracic and Vascular Surgery Department, Centre Hospitalier Universitaire Vaudois, University of Lausanne, Switzerland.

Disclosures: None.

M.G. and S.D. contributed equally to this report.

Received for publication April 11, 2010; revisions received May 13, 2010; accepted for publication May 27, 2010; available ahead of print June 30, 2010.

Address for reprints: Gonzalez Michel, MD, Thoracic and Vascular Surgery Department, Centre Hospitalier Universitaire Vaudois, University Hospital Lausanne, Rue du Bugnon 46, 1011 Lausanne, Switzerland (E-mail: Michel.Gonzalez@ chuv.ch).

J Thorac Cardiovasc Surg 2010;140:1198-9

$0022-5223 / \$ 36.00$

Copyright (c) 2010 by The American Association for Thoracic Surgery doi:10.1016/j.jtcvs.2010.05.041
}

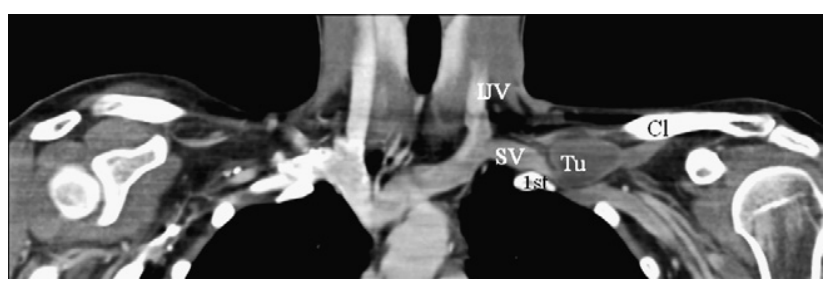

FIGURE 1. Cervical computed tomographic scan showing a left encapsulated hypodense mass of $3 \mathrm{~cm}$ in diameter, localized between the left clavicle and the first rib and compressing the middle portion of the subclavian vein. $I J V$, Internal jugular vein; $S V$, subclavian vein; $T u$, tumor mass; $C l$, clavicle; 1 st, first rib.

was necessary, taking out the subclavian vein, which was macroscopically adhering to the tumor until the level of the confluence with the left internal jugular vein (Figure 2). The subclavian artery and brachial plexus were dissected and preserved, with removal of all surrounding lymph nodes and fatty tissue. The thoracic duct was identified and ligated with nonresorbable sutures. The continuity of the venous axis was restored with division of the left internal jugular vein at the level of the carotid bifurcation and transposition to the axillary vein, with an end-to-end anastomosis using a 6-0 polypropylene running suture (Figure 3 ). Neither kinking nor stenosis was observed on the innominate vein. Histologic examination demonstrated a grade 2 myofibroblastic sarcoma with tumor-free margins. Owing to the presence of tumor cells near the margins, adjuvant radiotherapy was recommended. Except for a minimal chylothorax, which resolved after pleural drainage, the postoperative course was uneventful. The pain progressively decreased, the swelling disappeared, and the patient recovered full mobility. To avoid intimal hyperplasia, she was placed on a regimen of aspirin for antiaggregation for 1 year. At 1 month, duplex ultrasonography confirmed the patency of the anastomosis without stenosis. No local recurrence was observed at 12 months.

\section{DISCUSSION}

Tumors that involve the thoracic inlet, such as superior sulcus tumors, primary neurogenic tumors, soft-tissue neoplasms, or metastases, may invade the neural and vascular elements. ${ }^{1}$ Although their incidence is relatively low, surgical management can be difficult owing to a propensity toward local aggressiveness. However, only complete resection can offer acceptable long-term survival. ${ }^{1,2}$ Vascular 


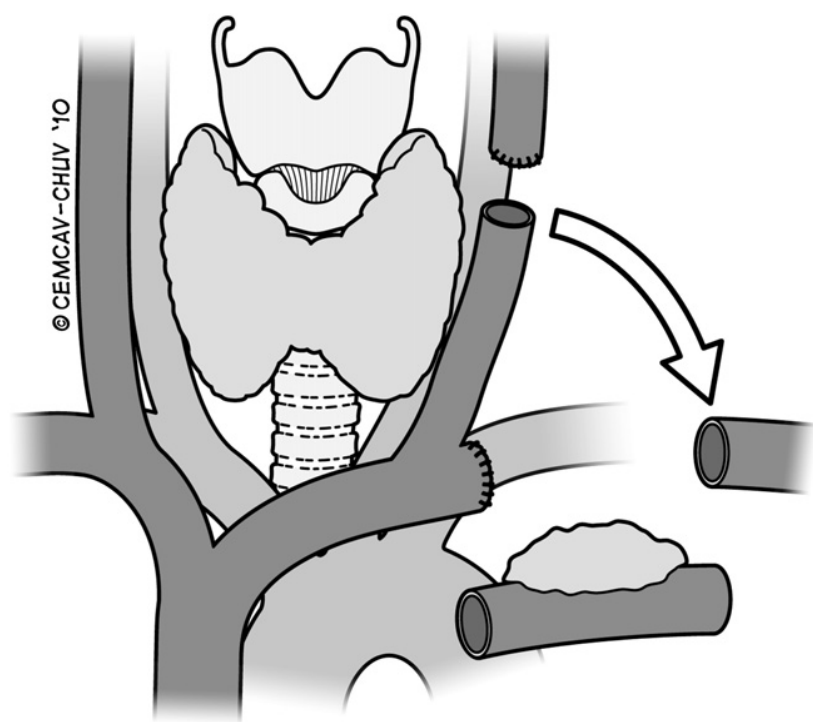

FIGURE 2. Schematic description of the technique of transposition of the internal jugular vein to the axillary vein after subclavian vein resection.

infiltration is frequently limited to the adventitial layer, allowing subadventitial dissection without resection. ${ }^{2}$ When the subclavian vein must be resected for oncologic reasons, reconstruction is rarely recommended. However, in some cases, especially in young patients with poor venous collateralization, revascularization should be considered to avoid sequelae of upper extremity venous hypertension, such as swelling. When the defect is short, the repair of the subclavian vein can be performed by direct anastomosis, but in the majority of cases, a bypass with interposition of an autologous vein or a polytetrafluoroethylene graft remains the standard procedure. ${ }^{2}$ In the setting of oncologic resection with lymphadenectomy and possible adjuvant radiotherapy, the risk of infection becomes significant, and venous material may be preferred in these cases. Nevertheless, morbidity associated with saphenous or femoral vein harvesting is not insignificant. However, owing to the localized fibrosis appearing after radiation therapy, polytetrafluoroethylene, providing the best patency rates in this situation, is often used. The problems of subclavian vein reconstruction are widely known in vascular access for hemodialysis. Various techniques have been proposed for the management of brachioaxillary-subclavian vein occlusion with upper limb arteriovenous fistulas or percutaneous dialysis catheters. ${ }^{3,4}$ Transposition of the internal jugular vein to the axillary vein was first described in 1966 for patients with subclavian vein thromboses related to hemodialysis access. ${ }^{5}$ Nevertheless, this technique has never been reported after subclavian vein resection for tumors invading the thoracic inlet, although the advantages of this technique are numerous: It is relatively easy to divide the internal jugular vein after an anterosuperior approach, and then only one single anastomosis

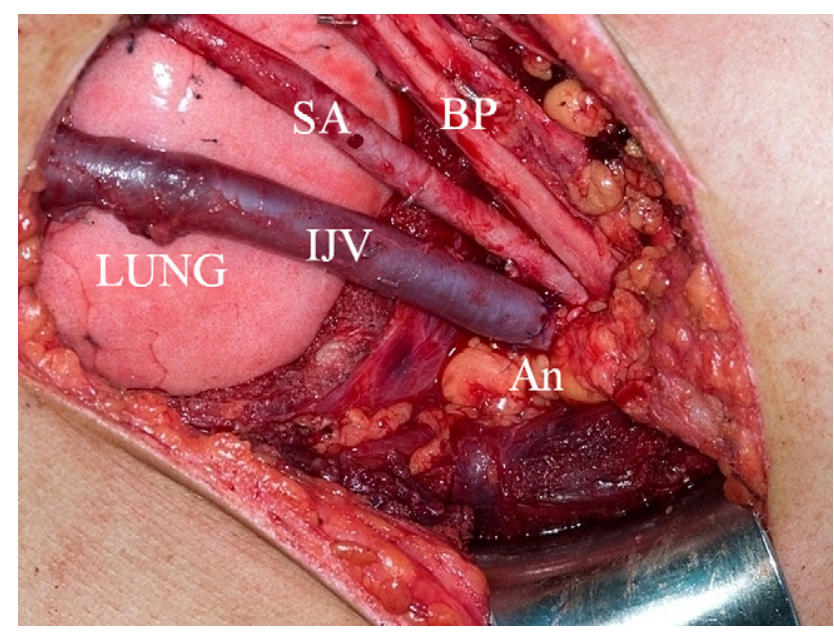

FIGURE 3. Intraoperative view after en bloc resection of the first rib, the left clavicle, and the subclavian vein. The subclavian artery and the brachial plexus have been dissected and preserved, but all fatty and lymphatic tissue have been removed. End-to-end anastomosis $(A n)$ between the internal jugular vein transposed and the axillary vein. $I J V$, Internal jugular vein; $S A$, subclavian artery; $B P$, brachial plexus.

must be performed, thereby reducing the operating time. The jugular vein is well matched in size to the axillary vein, facilitating anastomosis. There is no need for prosthetic material, considerably decreasing the incidence of infection. On the left side, the thoracic duct has to be recognized and individually ligated with care, thereby avoiding proximal injury to the ligated duct and an ensuing chylothorax. To prevent kinking at the innominate vein level, it is mandatory to exert gentle traction on the anastomosis. The patency of this technique has only been evaluated at 1 month, using duplex ultrasonography, which demonstrated no anastomotic stenosis. There are no guidelines for platelet antiaggregant therapy, but it seems reasonable to recommend aspirin for a minimum of 1 year to avoid intimal hyperplasia.

In conclusion, transposition of the internal jugular vein to the axillary vein seems to be an easy, safe, and effective technique that could be considered as a suitable alternative for subclavian vein repair, especially in young patients with poor venous collaterals.

\section{References}

1. Dartevelle PG, Chapelier AR, Macchiarini P, Lenot B, Cerrina J, Ladurie FL, et al Anterior transcervical-thoracic approach for radical resection of lung tumors invading thoracic inlet. J Thorac Cardiovasc Surg. 1993;105:1025-34.

2. Fadel E, Chapelier A, Bacha E, Leroy-Ladurie F, Cerrina J, Macchiarini P, et al Subclavian artery resection and reconstruction for thoracic inlet cancers. $J$ Vasc Surg. 1999;29:581-8.

3. Sottiurai VS, Lyon R, Ross C, Cooper M, Gonzales J. Surgical management of brachioaxillary-subclavian vein occlusion. Eur J Vasc Endovasc Surg. 1996;11: 225-9.

4. Gradman WS, Bressman P, Sernaque JD. Subclavian vein repair in patients with an ipsilateral arteriovenous fistula. Ann Vasc Surg. 1994;8:549-55.

5. Witte CL, Smith CA. Single anastomosis vein bypass for subclavian vein obstruction. Arch Surg. 1966;93:664-6. 